\title{
Neutronic analysis of the bi-spectral moderator such as that proposed for ESS
}

\author{
M. Magán ，F. Sordo ，L. Zanini ，S. Terrón ，A. Ghiglino ，F. Martínez \\ J.P. de Vicente , R. Vivanco , J.M. Perlado , F.J. Bermejo , F. Mezei , G. Muhrer
}

\begin{abstract}
A B S T R A C T
This paper reports on design studies concerning a moderator concept which aims to maximize the time averaged flux. The idea is to provide neutron spectra showing two clear maxima, with peaks at $(\sim 1 \AA)$ and $(\sim 2.5 \AA)$ arising from leakage from both cryogenic and thermal moderators. Such a concept known as a bi-spectral moderator (Mezei, 2006 [1]) while proven on a reactor source has only been examined for the ESS 2003 proposal. Filges et al. (2003 [2]), which featured a different design than the current ESS. This paper thus reports on a baseline design for such a moderator concept and shows that it can provide substantial gains in count rates for those applications not requiring high resolution in time-of-flight.
\end{abstract}

\section{Introduction}

As it is nowadays well established, neutrons generated by spallation reactions show a broad distribution of energies, with a low energy, $E_{n}<20 \mathrm{MeV}$ branch of cascade, evaporation and fission neutrons and a high-energy $E_{n}>20 \mathrm{MeV}$ band of spallation neutrons with energies as high as that of the impinging particles driving these nuclear reactions. The usefulness of such fast neutron beams is however limited to issues pertaining radiation physics of materials and devices as well as some applications in fields of Nuclear and Hadron Physics. In contrast to the typical energies of neutron beams suited for studies within the Condensed Matter Sciences are typically within the range of $0.1 \mathrm{meV}-$ $10 \mathrm{eV}$. This means that most neutrons have to be slowed down to usable energies within the epithermal, thermal or cold ranges by means of devices known as moderators, where neutron kinetic energies are brought down to values comparable to the thermal energy of particles comprising the moderator material. Efficient moderation thus involves repeated collisions with the moderator particles in which neutrons loose energy by transfer of energy and momenta to particles comprising the moderating media without being absorbed. The energy distribution of neutrons can be thus tailored by controlling the material out of which it is made as well as its size and temperature. It is worth recalling at this point that, practically any conceivable moderator will, because its finite thickness, contain an additional epithermal or slowing down component so that pulses leaking from the moderator surface comprise both a component of thermalized neutrons and an epithermal component.

Besides energy distribution, pulsed neutron sources require the spread in time of the neutron pulses leaving from the moderator to be as short as reasonably achievable since in terms of the source performance, one has to bear in mind that the achievable resolution attainable at the sample position of a given neutron scattering instrument operating on the time-of-flight principle, basically depends upon the spread in wavelengths of the incoming beam as roughly

$R=\Delta \lambda / \lambda$,

so that high-resolution applications would require pulses as narrow as possible, or alternatively, long secondary neutron flight-paths. To such an avail neutron absorbers or poisons have been deliberately added to the moderating media which yield the desired effect at the expense of a noticeable reduction in total flux.

Beam intensity has always been an issue for neutron scattering applications and therefore the quest to maximize this quantity has witnessed significant efforts to improve the performance to the 
target-moderator-reflector (TMR) complex alongside with concomitant increases in the source term. In fact, research into thermal and cold neutron production generated by high-energy protons is now a mature field of research which was inaugurated back in 1965 by Fraser et al. [3] who carried out absolutely normalized neutron yield measurements, validated a few years later at ORNL [4] and then at the Triumf Thermal Neutron Facility [5]. Of equal fundamental importance to the early developments was the absolutely normalized moderator spectra and pulse shapes of Graham's measurements-there was no equivalent to MCNP in those days [6]. Also paramount to pulsed-source developments is the reflector-moderator concept demonstrated at Argonne in 1972, which considerably enhances slow neutron intensities in all or most accelerator-based slow neutron sources. [See J. M. Carpenter "High intensity pulsed thermal neutron source", US Patent \# 3,778,627 (1973).]. Further work on moderator, coolant, and reflector was later published by Carpenter [7].

Also the British Spallation Neutron Source, later named ISIS came into being in late 1984 . Concurrently, a study phase for the German Spallations Neutronen Quelle carried out within the period 1980-1985 [8] motivated a variety of experiments on TMR systems which were performed at several accelerator laboratories such as PSI, CERN or the Saturne accelerator at Saclay (France) which provided a rich variety of results which paved the way to current developments carried out at last generation sources.

Maximization of the averaged neutron flux was soon identified as a must by a number of authors from Europe [9], the USA [10] and Japan [11]. An increase of intensity has thus been sought and found that it has oftentimes to be compromised against an adequate pulse structure in time, an issue that has lead to the quest for different arrangements depending upon the applications to be fed with such neutron pulses.

The purpose of the present work concerns the exploration of the performance of a moderator concept which in a way relaxes the strict pulse shaping criteria used so far by comprising both thermal and cold contributions to its energy spectrum. In doing so it aims to maximize the time averaged flux, a fact that may be of relevance for some applications. Such a concept, known as a bispectral moderator [1], has already been proven on a reactor source and it has also been already considered as an option for the ESS [2], although on a different design. The rationale behind such a construct, as schematically shown in Fig. 1 is to locate the neutron guides viewing parts of both cold and thermal moderators and in addition it is envisaged to employ materials such as Si wafers which are almost transparent to thermal neutrons to build those parts of the outgoing neutron guides facing the thermal moderator. A supermirror coating can thus be built using such material to

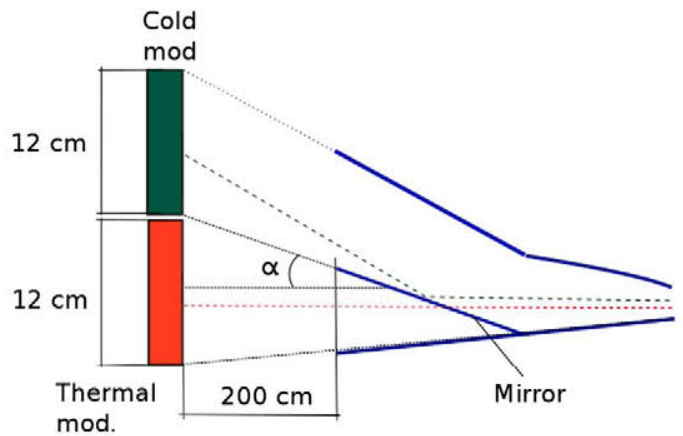

Fig. 1. A schematic drawing of the bi-spectral moderator concept. The proposal considers beam extraction by means of a twisted neutron guide comprising a Siwafer substrate. The guide part facing the thermal moderator behaves as an energy selector whereas the straight section is made of conventional neutron guide materials (see text). build those parts of the guides facing thermal neutrons so that its cut-off angle can be made to be proportional to the neutron wavelength and thus to provide some energy-selection of the outgoing thermal flux. It is thus the aim of this paper is to explore the optimum parameters for such a moderator to be used on the ESS long pulse source.

With such configuration, thermal neutrons freely cross into the guide through the mirror, whereas cold neutrons are reflected and transmitted into the same guide with high efficiency. This configuration allows to combine the spectra from two different moderators into the sample. As shown by Zendler [12], the transmission coefficient can reach up to $85 \%$ for $10 \AA$ neutrons and $95 \%$ for $<1.5 \AA$ neutrons, yielding a significant advantage over conventional extraction systems.

The European Spallation Source (ESS) project aims to build a next generation, accelerator-driven neutron source. Its baseline parameters are listed in Table 1 . One of its innovative features concerns the expected exploitation of such bi-spectral moderators. These moderators will provide two neutron spectra, one for the cold surface and another for the thermal surface, ideally showing two maxima, at energies $\sim 1 \AA$ and $\sim 2.5 \AA$, arising from neutrons leaked from cold and cryogenic moderators. Notice that, since the cross-section of para-hydrogen drops sharply at low energies, cold neutrons will not be completely moderated. Also, and due to the relatively low thickness of the water extensions, the spectrum emerging from the thermal extensions will not be completely thermalized. This is in agreement with previous studies [13].

The rationale behind such a moderator concept lies in the possibility for some instruments not requiring a fine selection of the incoming neutron energies to use both neutron spectra at the same time, thus leading to a significant enhancement in count rate, whereas instruments requiring higher resolution will view one of them only.

The basic target concept today under consideration banks on the design efforts carried out within the ESS project [13] as well as in concepts developed for the SNQ project [15] and a second target station (STS) at the Spallation Neutron Source at the Oak Ridge National Laboratory (SNS-ORNL) [16]. In particular, the target design nowadays under extensive design studies consists of a wheel containing tungsten elements cooled by helium with an average density which can reach values as high as $95 \%$ of the tungsten density and is kept rotating in the horizontal plane. The diameter of the wheel is $2.5 \mathrm{~m}$ in order to reduce the average heat load over the target material and to spread the radiation damage over a larger volume.

As it is well known from experience accumulated over the past four decades of operation of lower power spallation neutron sources, careful design of the systems aiming to reflect outgoing unmoderated or partially moderated neutrons as well as the moderators per se, are as critical for the final performance of the

Table 1

ESS baseline parameters as stated at the time of writing [14].

\begin{tabular}{llc}
\hline Parameter & Unit & Value \\
\hline Average beam power & $\mathrm{MW}$ & 5 \\
Number of target stations & & 1 \\
Number of beam ports & & 48 \\
Number of moderators & & 2 \\
Separation of ports in degrees & $\mathrm{GeV}$ & 5 \\
Proton kinetic energy & $\mathrm{mA}$ & 2.5 \\
Average macro-pulse current & $\mathrm{ms}$ & 50 \\
Macro-pulse length & $\mathrm{Hz}$ & 2.86 \\
Pulse repetition rate & $\mathrm{MV} \mathrm{m}{ }^{-1}$ & 14 \\
Maximum gradient for superconducting linac & $\mathrm{m}$ & 40 \\
Maximum linac length & $\mathrm{h}$ & 482.5 \\
Operating hours/year & & 5200 \\
\hline
\end{tabular}


installation as the neutron generating target as such. Here we present some calculation details pertaining the candidate moderators to be installed at the ESS target station. A basic moderator geometry as well as the optimum moderator material are selected on the basis of calculations described in the next section. An iterative optimization scheme is then applied to find optimal values of the main parameters. Finally some calculations are also reported on the sensitivity of the moderator performance to details of its molecular composition, namely the ortho-para ratio of a hydrogen moderator and also the usefulness of installing a cooled beryllium filter close to the moderator viewed surface is discussed.

\section{Methodology for moderator analysis}

The accelerator parameters listed in Table 1 impose severe constraints to the moderator configurations that need to be considered in detail. First, the final proton energy of $2.5 \mathrm{GeV}$ precludes the use of any moderator configuration with a direct view of the target to prevent leaking out of large fast neutron backgrounds. This leaves the moderator WING configuration as the only one practicable, since implementation of other configurations such FLUX-TRAP becomes difficult to conceive for a rotating target. Second, the long proton pulses which are well in excess of the moderation time, preclude tailoring of the neutron time distribution by recourse to standard decoupling procedures to the moderator as done in already existing facilities. As the time distribution of neutrons is practically set by the pulse length, the moderator design should aim at maximization of the outgoing flux. To do so, a coupled moderator with a large volume appears as the best choice [17].

Other considerations are also of relevance, particularly concerning the moderator material and its precise location with respect to the target element. Solid methane, a widely used material for the purpose seems precluded since from previous experience [18] it is estimated that due to the expected radiation levels its life span would be of just of a few minutes. Liquid parahydrogen in turn, remains at the time of writing as the most promising material for the purpose. Also, because of the need to locate 48 beam ports $2 \mathrm{~m}$ away from the moderator, this forces the use of two wing moderators, each one with two $60^{\circ}$ openings in the reflector.

To proceed with the design, we have profited from experience gained at the Materials and Life Science Experimental Facility (MLSF) hosted by the KEK and JAEA within the J-PARC collaborative project. There, a cold coupled para-hydrogen moderator is operative [19]. Also, studies carried out by F. Gallmeier for the Second Target Station project at the Spallation Neutron Source (STS-SNS) at the Oak Ridge National Laboratory [20] provided valuable data and insights for our purposes.

From considerations explained above we have developed a geometrical design which consists of a cylindrical para-hydrogen moderator with a light water premoderator. The moderator viewed window is $12 \mathrm{~cm}$ high with a total viewed surface of $144 \mathrm{~cm}^{2}$. Both the moderator and premoderator are coupled to a $75 \mathrm{~cm}$ radius and height beryllium reflector cooled by heavy water in order to maximize the neutron flux. Finally, the target-moderator-reflector system is surrounded by $4 \mathrm{~m}$ of iron shielding.

In a bi-spectral system such as that depicted in Fig. 1, a neutron mirror is positioned $2 \mathrm{~m}$ away from the surface of the moderators, making an angle $\alpha$ to the normal of the moderator surface. This angle matches the critical angle for which cold and thermal moderator flux become of equal intensity, which corresponds to a cross-over wavelength $\lambda_{v}$. In real numbers, for a neutron guide with $m=5$, such angle is $1.175^{\circ}$ [12]. As shown in Introduction, this system allows superior beam extraction efficiency.
As already referred to above, the concept of bi-spectral extraction requires two viewed surfaces, cold and thermal, with as separation as small as possible between them. From a practical point of view, such a concept can be implemented on a cylindrical cold moderator by adding two $12 \mathrm{~cm}$ long light water extensions (Fig. 2) to the premoderator. Such extensions make up a viewed surface of $144 \mathrm{~cm}^{2}$ for the thermal spectrum. These elements slightly decrease the cold moderator brightness due to the reduction of the reflector volume around it. Nevertheless, and from a global point of view, the gain in thermal neutrons overweights this loss.

Notice that, because of the relative position of the view windows with respect to the neutron beam, the thermal extensions are not placed symmetrically with respect to the neutron production peak, and, therefore, they must be analyzed separately. As shown in Fig. 2, we shall name Thermal Moderator 1 as the surface placed further along the proton beam direction and Thermal Moderator 2 as the surface placed nearer.

In order to proceed with the optimization, a parametric MCNPX model has been developed (Fig. 2A and B). The optimization of the different parameters has been carried out stepwise, changing one of the parameters and keeping the others constant. Changing values for the main parameters (moderator radius, moderator position and reflector size) leads to changes in brightness which are largely independent so that minimal differences at the final configuration could be found by changing the order of the optimization process. We have used MCNPX 2.7e, using the Intel Fortran Compiler and OpenMPI for parallelization.

Regarding the employed nuclear cross-sections, the latest edition of the Los Alamos National Laboratory scattering kernel is used in this paper [21] together with the ENDEF-B/VII crosssection libraries [22]. There are several isotopes for which proton cross-sections are not included in this data library. In such cases,

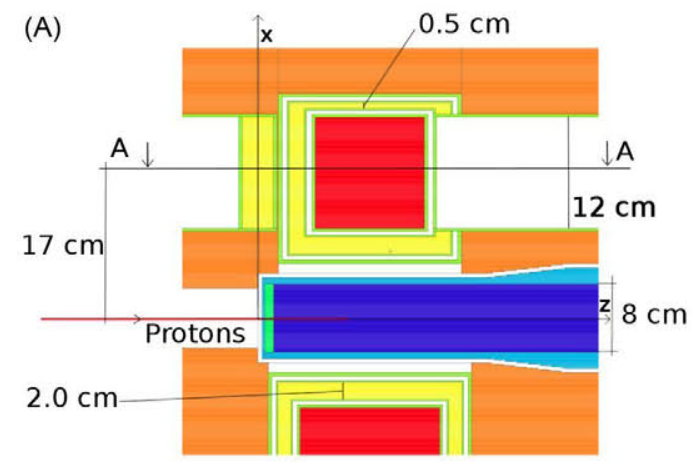

(B)

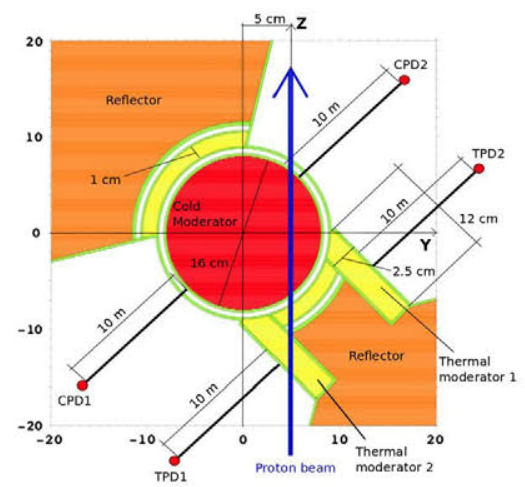

Fig. 2. The figure depicts the schematic model employed in calculations using MCNPX. The upper frame $2 \mathrm{~A}$ shows a horizontal cross-section of the targetreflector-moderators complex, showing the most relevant dimensions. The lower frame $2 \mathrm{~B}$ shows some of the details pertaining the configuration of point detectors employed to monitor the neutron emission time distributions. 
TENDL-2010 [23] has been used. For high energy reactions (above $20 \mathrm{MeV}$ ), the intranuclear cascade model CEM [24] is applied.

Neutron emission time distributions have been evaluated by means of four point detectors located at positions shown in Fig. 2A. There are two detectors for thermal neutrons (TPD1 and TPD2) and another two for cold neutrons (CPD1 and CPD2), symmetrically positioned at $10 \mathrm{~m}$ from the moderator surfaces. Notice, however, that such detectors are not positioned symmetrically with respect to the incoming proton beam and therefore, full symmetry of the outgoing neutron flux leaking from the moderator cannot be expected. The point detectors were enclosed within collimators to avoid indirect contributions. Neutron timeof-flight from the moderator surface to the detector is subtracted in order to have the correct time distribution on the moderator surface [25].

At this point, it is worth emphasizing that moderator performance is sensitive to target configuration [26], which means that modifications in the target design could alter the results presented in this study. Nevertheless, the conclusions of previous studies have shown that the target selection should not be determined by neutronic optimization because differences between target concepts are not dramatic. Hence, the main conclusions of this document should remain valid for different target options.

\section{Optimization process for the moderator}

The model previously described has been used in a parametric optimization process. Within it, each of the system parameters has been systematically scrutinized, until an optimal configuration has been reached. A set of 10 variables have been taken into account, considering as the main figure of merit the cold neutron flux $(<5 \mathrm{meV})$ in the cold moderator surface. Nevertheless, the thermal and cold flux $(<431 \mathrm{meV})$ in the premoderator extensions have also been scored in order to analyze the evolution of thermalneutron brightness. Notice however that optimization has been carried out within a single-parameter space and therefore, the cross-interaction between parameters has not been quantified.

The first parameters considered in the optimization are the radius of the cold moderator and the thickness of the water thermal extensions. Fig. 3 shows the dependence of the brightness of cold neutrons with the radius of the cold moderator. The graph depicts a broad maximum for values between $8.5 \mathrm{~cm}$ and $10 \mathrm{~cm}$. In addition, varying the radius of the cold moderator does have an effect on the brightness of thermal neutron beams since it

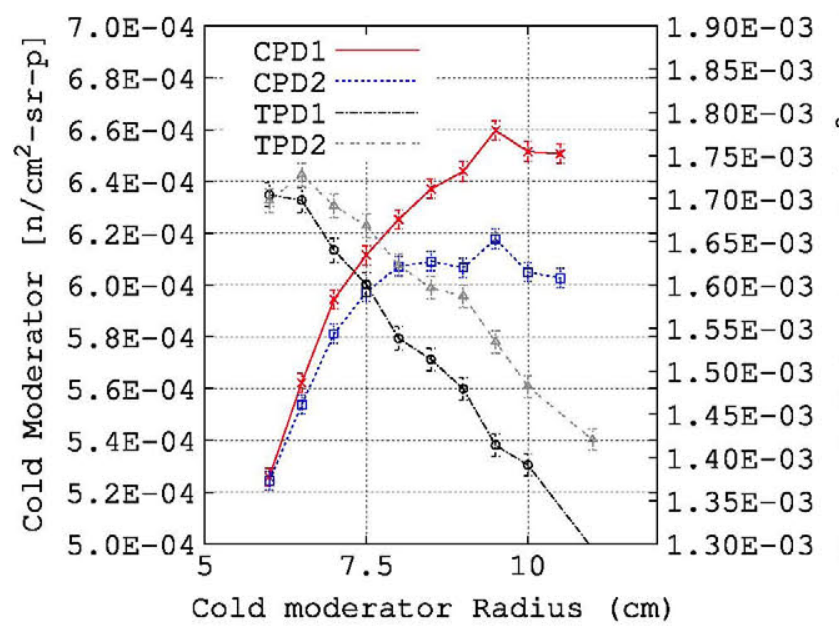

Fig. 3. Neutron brightness as estimated by the two cold neutrons (CPD1, CPD2) and two thermal neutrons (TPD1, TPD2) point detectors for different values of the radius of the cold moderator. translates into an increase of distance to the neutron production maximum. The selected value for the moderator radius was then set to $8 \mathrm{~cm}$, which is not far from optimal values for cold neutron production, and somewhat mitigates the deleterious effect that larger values for the radius have on the thermal-neutron flux.

As far as the water extensions are concerned, Fig. 4 shows the dependence of neutron brightness on the thicknesses of such extensions. A glance to the figure shows that brightness for thermal neutrons reach optimum values within the $1.6-2.85 \mathrm{~cm}$ interval while cold neutron fluxes are, as expected, unaffected. Further calculations were performed, and the thermal neutron spectra were analyzed for the two extreme values of the interval just quoted. The spectrum resulting from thicker extensions is slightly better thermalized, as shown in Fig. 5, and on such grounds a value of $2.85 \mathrm{~cm}$ was chosen as the optimal thickness.

Fig. 6 shows the dependence of moderator brightness on the $Z$-axis position, or relative position between target and moderator as measured by the horizontal displacement of the moderator center with respect to the point where the proton beam hits the target wheel. Notice that the positions of the thermal-moderator extensions are not symmetric with respect to the beam footprint over the target, which explains why their sensitivity to the relative position of moderator to target is not the same. For the cold moderator, maximal flux values are reached within an interval of $8-12 \mathrm{~cm}$. In contrast the thermal-neutrons extensions show a

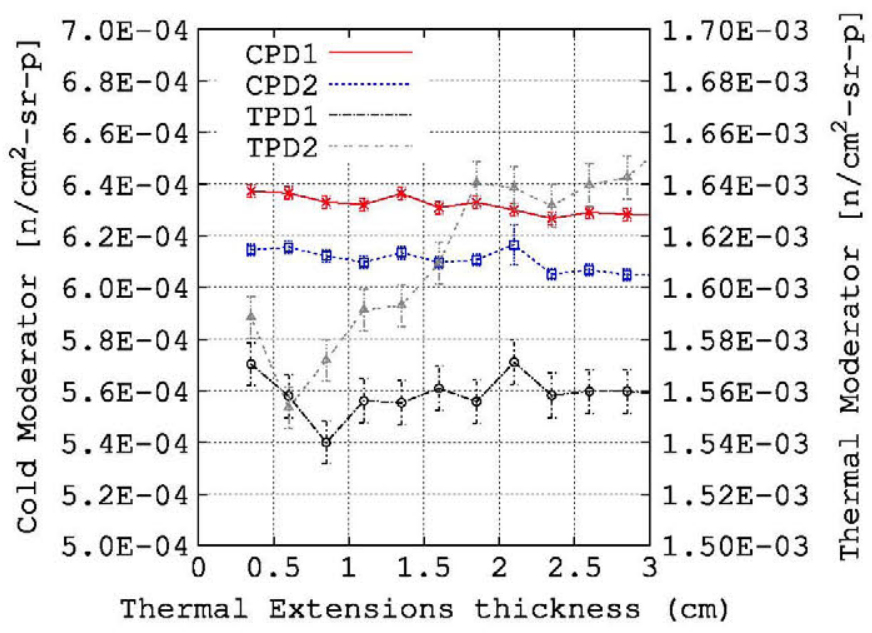

Fig. 4. Neutron brightness as estimated by the two cold neutrons (CPD1, CPD2) and two thermal neutrons (TPD1, TPD2) point detectors for different values of the thickness of the water extensions.

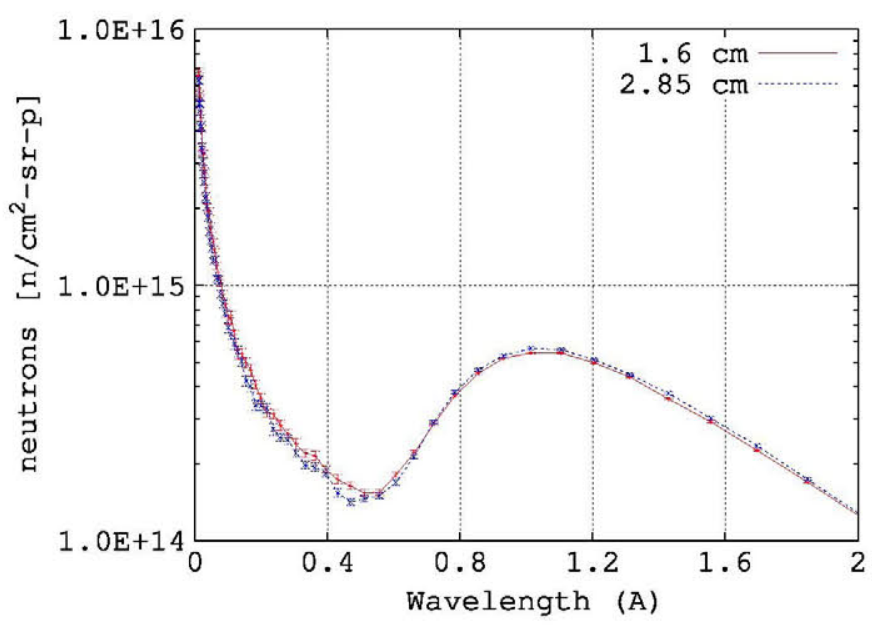

Fig. 5. Thermal neutron spectra leaking the moderator for 1.6 and $2.85 \mathrm{~cm}$ thick thermal extensions. 


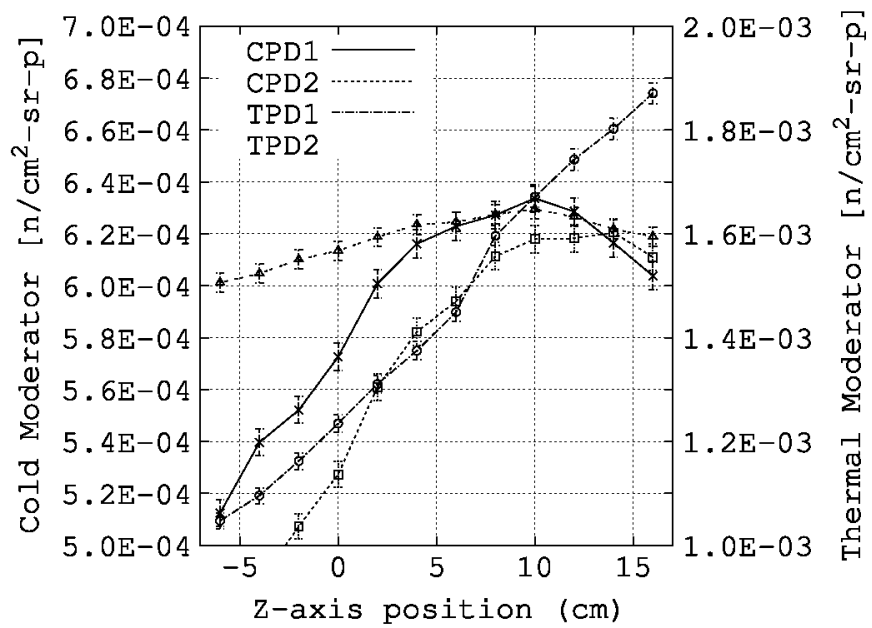

Fig. 6. Evolution of neutron brightness with the offset between target and moderators.

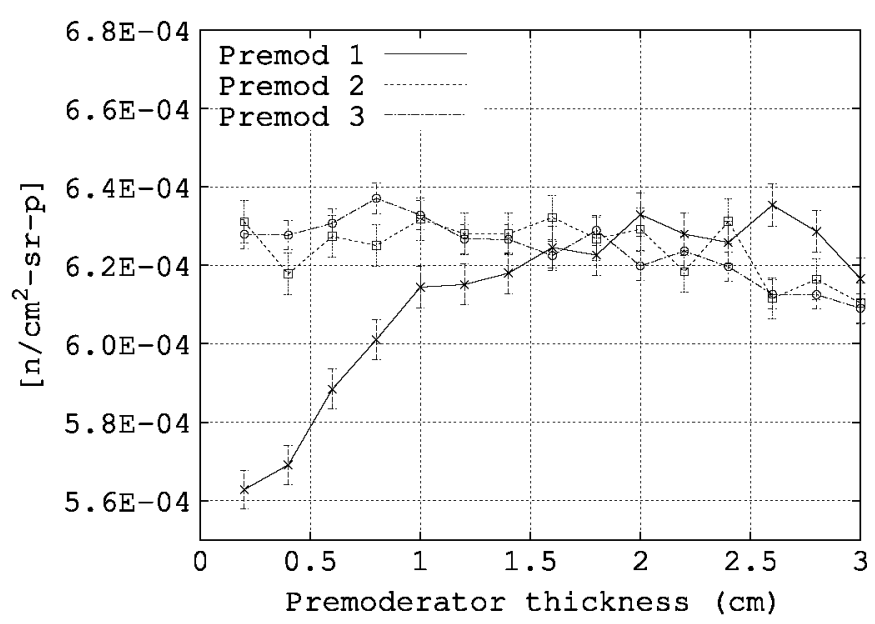

Fig. 7. Dependence of the cold neutron brightness on the premoderator thicknesses.

broad profile centered at $10 \mathrm{~cm}$ in one case and a continuous rise in the second. Choosing a distance of $10 \mathrm{~cm}$ between moderator and target thus shows a good balance between both thermal extensions. Regarding the cold moderator, locating it at $10 \mathrm{~cm}$ comes close enough to the maximum.

The premoderator role is to decrease the energy of neutrons before entering the moderator. This results in an increase of the efficiency of the cold moderator and also, reduces the heat load. The energy spectrum of neutrons coming out from the target is different than the spectrum of those coming from the reflector, so the optimum thickness of the premoderator facing the target (premoderator 1) will be different from the optimal thickness of those facing the reflector (premoderators 2 and 3). Fig. 7 shows the dependence of cold neutron brightness on the premoderator dimensions. The results depicted in the figure show that, as far as the premoderator facing the target is concerned, there is a plateau between $1.5 \mathrm{~cm}$ and $2.5 \mathrm{~cm}$ values for the premoderator thickness. However, larger premoderators do have the advantage of lower heat generation, as shown in Fig. 8, and thus, while not offering an improvement in performance, they do lower the costs of the cryogenic system. In fact, a variation from 1.4 to $2.7 \mathrm{~cm}$ in premoderator 1 thickness allows a reduction of the average heat load in the cold moderator from 6800 to $5500 \mathrm{~W}$. Therefore, on such grounds we have set the optimum thickness to $2.7 \mathrm{~cm}$ for the premoderator area facing the target. For the areas facing the reflector, the effect is very small, and the optimum is $1 \mathrm{~cm}$.

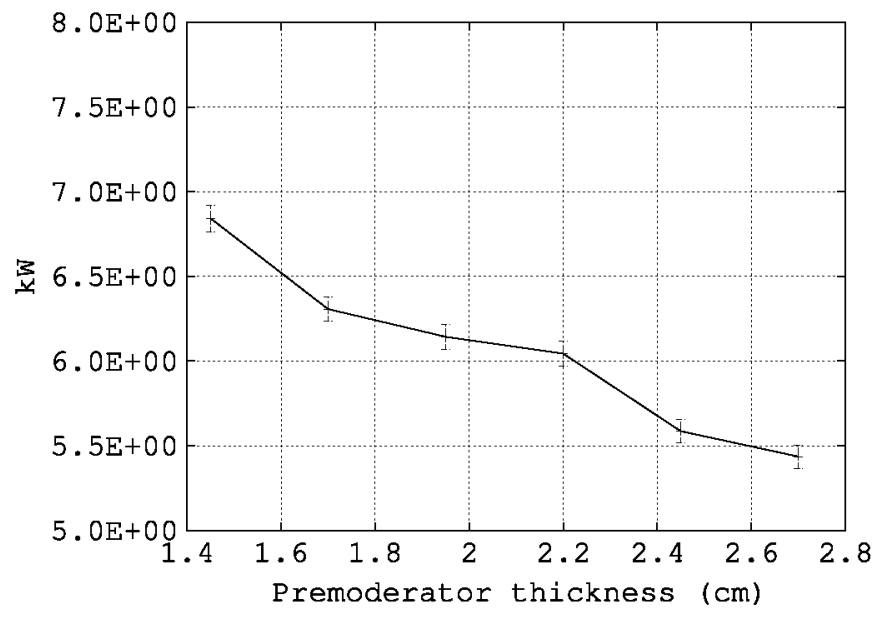

Fig. 8. Dependence of the heat deposited in the cold moderator with the thickness of premoderator 1.

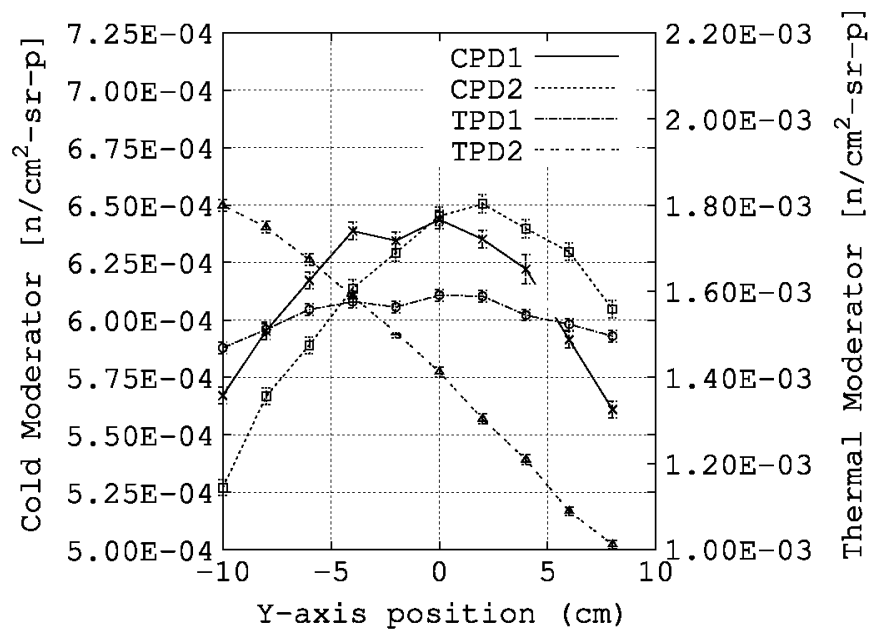

Fig. 9. Dependence of thermal and cold neutron fluxes on the displacement of the moderators along the $Y$-axis.

Finally, the $Y$-axis position, or relative position of the moderator with respect to the beam center has also been optimized. If the position of the cold moderator is chosen to be symmetrically placed around the beam $(Y=0)$, the thermal extensions are then placed far from the neutron production peak, decreasing their performance. Fig. 9 shows the cold and thermal neutron brightness when changing the moderator displacement along the $Y$-axis. A small displacement of $5 \mathrm{~cm}$ in the negative $Y$-direction reduces cold neutron production by only $5 \%$, while yielding a good balance between both thermal extensions. This balance between extensions could also be achieved by choosing a different angle between beam entrance and experimental windows. Nevertheless these angles are finally set by guide inserts feeding the instruments as well as by rotating-target constraints. Taking into account the displacement in the beam direction and this normal displacement, the final position of the peak of neutron production is close to the area in between cold moderator and thermal extensions.

\section{Reflector material analysis}

The studies carried out at J-PARC [27] show that a reflector made of lead performs better than beryllium in short pulse operation if analyzed in terms of neutron pulse peak width and 
decay time but yields a lower time integrated flux. This conclusion could change with the ESS proton pulse parameters. In order to clarify this issue several materials have been taken into consideration, which apart from lead are beryllium, light and heavy water. Iron is another possibility not examined here.

The main nuclear properties of beryllium as a reflector material are its significant moderation capability, its very low threshold for $(n, x n)$ reactions, and a relative low capture cross-section. Lightwater in turn offers high moderating power, but on the other hand it has also a significant neutron absorption cross-section and very limited neutrons produced by $(n, x n)$ reactions compared to Be. Lead has the advantage of its high density and its low absorption cross-section, but it does not moderate neutrons. Alternatively, heavy-water provides low neutron absorption and significant moderation but, as a drawback, it has very few neutrons produced by $(n, x n)$ reactions compared to $\mathrm{Be}$.

In order to assess the performance of candidate reflector materials, an optimization of the radius and height parameters has been performed for each one of them. The results are qualitatively in line with what could be expected according to the previous paragraph. The optimum radius and height found for the cylindrical shape reflectors are equal, as the problem is quite isotropic around the moderator, and their values are: $30 \mathrm{~cm}$ for light water, $75 \mathrm{~cm}$ for lead, $65 \mathrm{~cm}$ for the heavy water and $75 \mathrm{~cm}$ for beryllium. Based on the optimal dimensions for each material, a pulse shape analysis is summarized in Table 2 .

The signal considered here corresponds to the integral value up to the proton pulse length $(2.857 \mathrm{~ms})$ and the tail is the integral from the end of the proton pulse to the end of the simulation time. The signal value shows that the beryllium reflector has a performance around $10 \%$ better than the lead reflector, but the tail distribution is significantly longer. As an additional way to measure the pulse shape, the table shows the time required for each pulse to drop to $50 \%$ and $10 \%$ of the peak value. Using a beryllium reflector, the pulse decays much slower than using lead or water, and it will take twice longer to decrease down to $10 \%$ of the peak value.

\section{Sensitivity to ortho/para ratio}

Hydrogen molecules within their vibrational ground state exist in different quantum states which arise from complete quantization of the rotational wavefunction. The rotational ground state corresponds to para-hydrogen which corresponds to a singlet configuration with total nuclear spin $\mathrm{I}=0$ for its ground state and even values for its rotationally excited levels. On the other hand, the triplet ortho-hydrogen has $\mathrm{I}=1$ and odd values for its rotational levels. At temperatures of about $20 \mathrm{~K}, 99.9 \%$ of a hydrogen sample in thermal equilibrium will correspond to para- $\mathrm{H}_{2}$. A transition para $\rightarrow$ ortho $-\mathrm{H}_{2}$ can be induced by the neutron magnetic moment provided that its energy exceeds the $14.5 \mathrm{meV}$ of such a spectroscopic rotational transition. For a spallation source, these two states present a critical difference in their function as a moderator: for neutron energies below approximately $10 \mathrm{meV}$, the para-hydrogen cross-section is unusually low, almost two orders of magnitude lower than orthohydrogen cross-section, which follows a more conventional $\sim 1 / v$ pattern [28]. This means that the concentration ratio of these states can have a large impact on cold neutron brightness, and therefore the ratio of para/ortho needs to be critically assessed.

At the cryogenic temperatures projected for ESS (18-25 K), the composition in thermal equilibrium is less than $1 \%$ ortho-hydrogen, but irradiation conditions do significantly increase this figure. Catalyst systems, in turn, can convert ortho-hydrogen back to para-hydrogen. While at the operation temperatures considered, hydrogen spontaneously turns back to the para-hydrogen state, the process which involves molecular collision energy-transfers is extremely slow without the use of catalysts [29]. So far, there is still no estimation of expected ortho/para ratios at ESS, so the effect of ortho-hydrogen concentration up to $75 \%$, which is the percentage corresponding to normal hydrogen, is explored, in order to estimate the potential effects of ortho-hydrogen presence.

Using the geometry described in previous paragraphs, simulations were performed with different moderator compositions, particularly with increasing concentration of ortho-hydrogen. Table 3 summarizes the results, using the same metrics as those employed in the previous section. Increasing the ortho-hydrogen concentration slightly reduces the signal to noise ratio (around $2 \%$ per $5 \%$ of ortho-hydrogen), and slightly increases decay time. This means that not only the brightness is reduced by significant concentrations of ortho-hydrogen, but also the pulse quality deteriorates, so that a catalysis system to ensure a concentration of para-hydrogen as close as possible to its equilibrium value will be required. These results are also in agreement with those obtained by other researchers [30].

\section{Beryllium filter}

Metallic Beryllium if cooled below $80 \mathrm{~K}$ is almost transparent to neutrons with energies below $5 \mathrm{meV}$. This effect could be exploited to increase the moderator performance by covering the cold neutron transport lines with cold beryllium, in the same way as done at Los Alamos Neutron Source [31]. This system will reflect

\section{Table 3}

Neutronic performance for moderators composed of different ortho-hydrogen concentrations. Notice that while under equilibrium conditions and temperatures expected to operate, liquid hydrogen contains a fraction below $1 \%$ of the rotationally excited ortho species, the composition right after condensation from room-temperature gas contains about $25 \%$ of such species.

\begin{tabular}{lllll}
\hline Parameter & Pure para & $10 \%$ Ortho & $25 \%$ Ortho & $75 \%$ Ortho \\
\hline Signal $\left(\mathrm{n} / \mathrm{cm}^{2}-\mathrm{eV}-\mathrm{sr}-\mathrm{MW}\right)$ & $2.54 \times 10^{13}$ & $2.31 \times 10^{12}$ & $1.99 \times 10^{13}$ & $1.50 \times 10^{13}$ \\
Tail $\left(\mathrm{n} / \mathrm{cm}^{2}-\mathrm{eV}-\mathrm{sr}-\mathrm{MW}\right)$ & $3.66 \times 10^{12}$ & $3.44 \times 10^{12}$ & $3.07 \times 10^{12}$ & $2.49 \times 10^{12}$ \\
Ratio & 6.94 & 6.7 & 6.49 & 6.01 \\
Decay time to $50 \%(\mu \mathrm{s})$ & 180 & 200 & 210 & 230 \\
Decay time to $10 \%(\mu \mathrm{s})$ & 890 & 900 & 930 & 1010 \\
\hline
\end{tabular}

Table 2

Neutron pulse shape metrics for the different reflector materials with optimal size.

\begin{tabular}{|c|c|c|c|c|}
\hline Parameter & $\mathrm{Be}$ & $\mathrm{Pb}$ & $\mathrm{D}_{2} \mathrm{O}$ & $\mathrm{H}_{2} \mathrm{O}$ \\
\hline Peak (n/cm $\left.{ }^{2}-M W-\AA-s r-s\right)$ & $1.92 \times 10^{13}$ & $1.76 \times 10^{13}$ & $1.53 \times 10^{13}$ & $1.18 \times 10^{13}$ \\
\hline Signal (n/ $\left.\mathrm{cm}^{2}-\mathrm{MW}-\AA-\mathrm{sr}\right)$ & $4.72 \times 10^{10}$ & $4.66 \times 10^{10}$ & $3.76 \times 10^{10}$ & $3.1 \times 10^{10}$ \\
\hline Tail (n/cm $\left.\mathrm{cm}^{2}-\mathrm{MW}-\AA-\mathrm{sr}\right)$ & $8.25 \times 10^{9}$ & $3.59 \times 10^{9}$ & $6.63 \times 10^{9}$ & $2.85 \times 10^{9}$ \\
\hline Signal to tail ratio & 5.72 & 13.0 & 5.67 & 10.8 \\
\hline Time to $50 \%$ decrease $(\mu \mathrm{s})$ & 180 & 130 & 170 & 160 \\
\hline Time to $10 \%$ decrease $(\mu \mathrm{s})$ & 1050 & 410 & 1070 & 520 \\
\hline
\end{tabular}




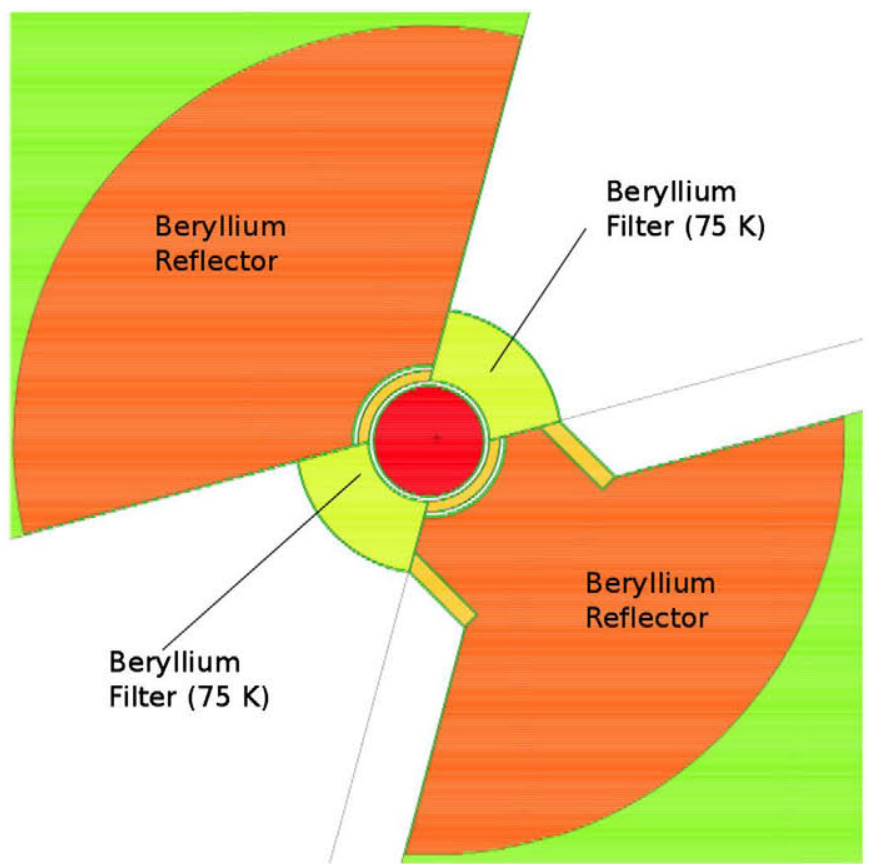

Fig. 10. Beryllium filter geometry considering a filter width of $11 \mathrm{~cm}$.

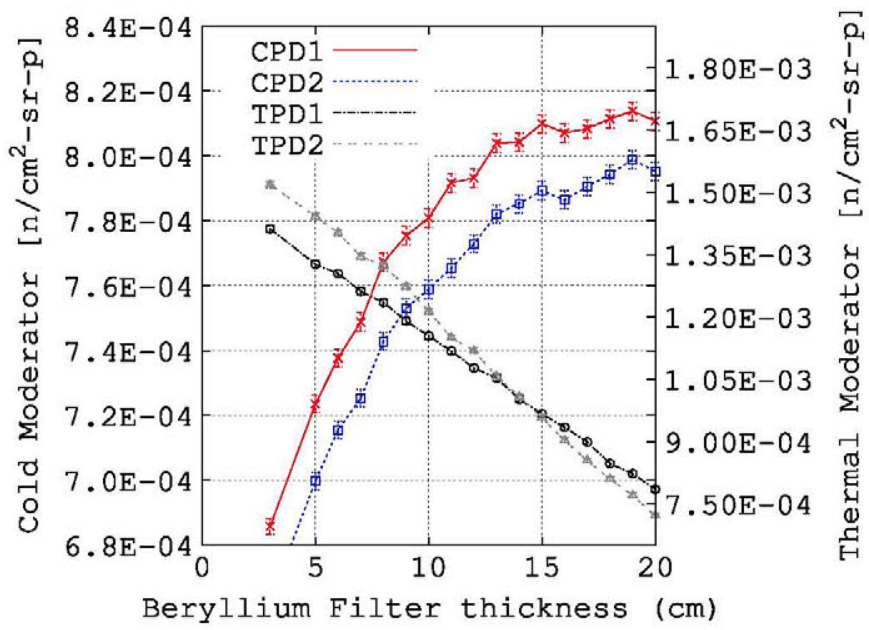

Fig. 11. The effect of the thickness of the beryllium filter on the neutron beam brightness for cold neutrons having energies less than $5 \mathrm{meV}$ and beams with energies below $400 \mathrm{meV}$.

thermal and fast neutrons back into the moderator, while allowing neutrons within the cold range to pass through, increasing neutron flux over the moderator. However, cold beryllium will have a significant cross-section for thermal neutrons, and therefore, thermal extensions have to be placed in the border of the filter as it is shown in Fig. 10 in order to keep the filter out of the path of thermal neutrons. Doing so, however, will cause the thermal extensions to be displaced from the neutron production maximum, reducing their performance.

Fig. 11 shows the effect of the filter on the cold and thermal surfaces based on the scattering kernels for cold beryllium provided by G. Muhrer [32]. For the cold moderator, a continuous increase of the flux up to a plateau is observed. This plateau is about $12 \%$ higher than the reference value without a filter, and it is reached at $20 \mathrm{~cm}$ thickness. At the same time, a continuous decrease of the thermal performance is observed due to the displacement introduced by the filter to the extensions. This geometrical effect could be partially avoided if part of the instruments were allowed to see only cold neutrons. However, design restrictions at the time of writing discard this possibility, leading to a $60 \%$ decrease in estimated thermal performance.

In summary, a beryllium filter will result in an increase of cold neutron production from the bi-spectral moderator by $12 \%$ at the expense of a $50 \%$ decrease of thermal neutrons. Hence, a beryllium filter is discarded for this preliminary analysis. Nevertheless, it merits to be considered for those neutron lines where the use of the bi-spectral moderator is not foreseen.

\section{Discussion}

The results of the calculations reported here show that a parahydrogen moderator is the best choice on the grounds of moderating efficiency, good temperature homogeneity and the absence of large radiation damage effects to implement the bi-spectral concept.

The proposed concept for a bi-spectral moderator consists, apart from the thermal moderator, of a moderator structure comprising a cylindrical, main cold moderator body plus two $12 \mathrm{~cm}$ long light water extensions to the premoderator as shown in Fig. 2. Such an arrangement provides a viewed surface of the thermal spectrum of some $144 \mathrm{~cm}^{2}$. Addition of such light-water extensions leads to a small decrease of brightness for the cold moderator due to a reduction of the reflector volume around it. Such an effect is however compensated by the 10-fold gain achieved in thermal neutrons flux.

Considering the bi-spectral configuration described on previous paragraphs a complete evaluation of the pulse shape for the ESS cold moderator has been performed. Fig. 12 shows the pulse shapes on the cold moderator surface for different wavelengths and Fig. 13 shows cold and thermal neutron spectra on the moderator surfaces for the bi-spectral moderator.

As emphasized several times in the relevant literature [34] a comparison of the expected ESS performance with that of the reactor at the ILL cannot sensibly be made using a single figure of merit, since chosen parameters such as the time-averaged total flux or the peak flux can be grossly misleading if used alone. In fact, while in terms of time averaged source brightness figures typical of the ILL of $450 \times 10^{10} \mathrm{n} / \mathrm{cm}^{2}$ s sr $\AA$, which surely outdo the figure expected for ESS, the peak fast neutron production of the latter will surely exceed the reactor average value by two orders of magnitude. At any rate, any meaningful comparison would have to consider the performance of both pulsed and reactor sources at a given wavelength. With such provisos in mind we show in Fig. 13 a

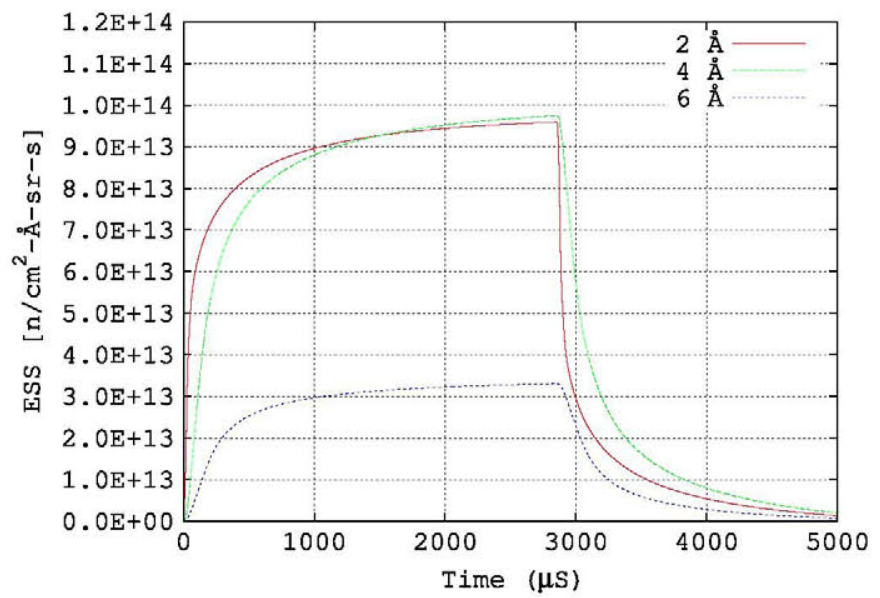

Fig. 12. Pulse shape for $14 \mathrm{~Hz} @ 2.857 \mathrm{~ms} @ 5 \mathrm{MW}$ at 2,4 and $6 \AA$. 
(A)

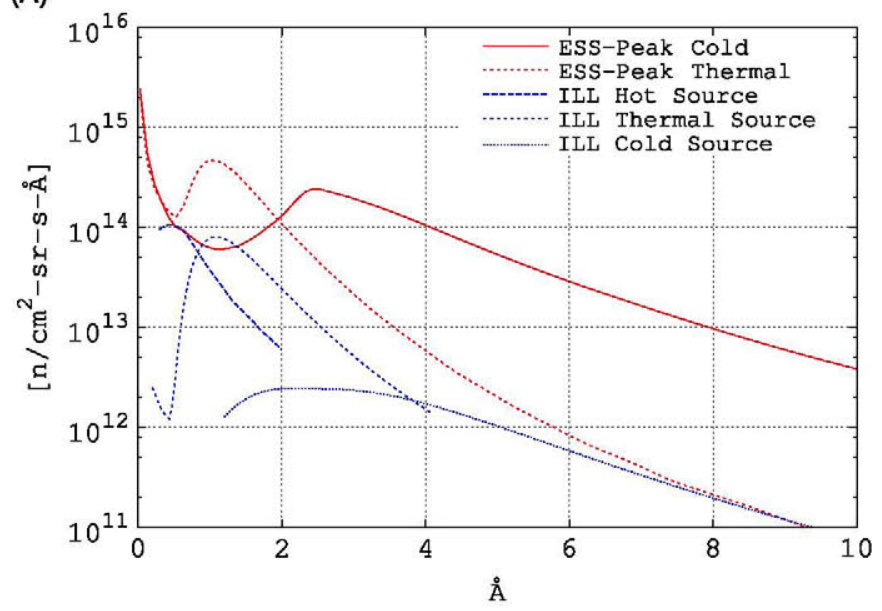

(B)

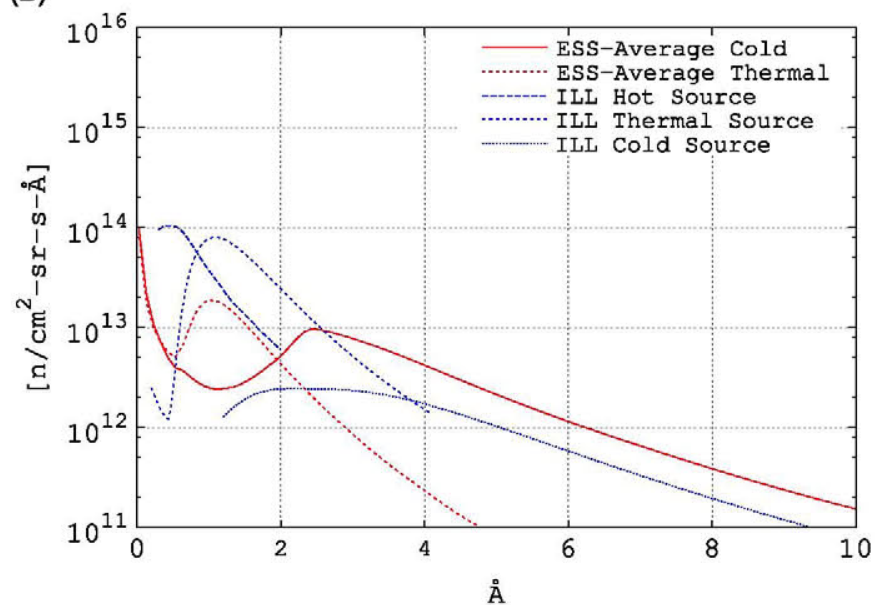

Fig. 13. The upper frame shows the expected peak neutron flux for ESS $(14 \mathrm{~Hz} @$ $2.857 \mathrm{~ms} @ 5 \mathrm{MW}$ ) compared to the time averaged figure measured at the ILL [33]. The lower frame compares the average neutron flux expected for ESS $(14 \mathrm{~Hz}$ @ $2.857 \mathrm{~ms} @ 5 \mathrm{MW}$ ) compared to the same quantity as measured at the ILL [33].

comparison of the expected performance of ESS as measured by its peak neutron flux to the time average flux of the ILL reactor [35], as well as the time averaged source brightness of both facilities. The relevant issue of such a comparison concerns the expectation of large numbers of long wavelength (i.e. greater than $6 \AA$ ) neutrons coming out of the pulsed source, which favorably compares to those resulting from the ILL cold deuterium moderator.

\section{Summary}

As final conclusions from the analysis carried out concerning the geometrical variables that configure the source, we have made some compromises to keep a reasonable balance between thermal and cold neutron performance. Also, some of the parameters, while having scant influence on the neutronics, do have an effect on heat generation, and therefore this aspect had to be considered in sufficient detail in the optimization of some of them.

To the best of our knowledge, para-hydrogen appears as the best cold moderator materials, both on neutronics and engineering grounds. On the other hand, as far as the thermal moderator is concerned, the proposed thermal extensions cause a small loss of cold brightness, but the 10-fold increase in thermal flux produced as a result of their explicit inclusion, overweights this loss and results in a more useful source.
The summary analysis of options concerning reflector materials shows that beryllium is the best choice since it maximizes neutron production. However, use of a beryllium filter as tested at installations like LANSCE does not seem advisable for those extraction lines feeding high resolution instruments, since the significant loss of thermal flux is not at all compensated by the gain in cold neutron brightness. Notice however that most of the high-resolution instruments which will view the cold moderator will surely benefit from such a filter device.

As regards the optimal choice of a moderator material to provide cold neutrons, para-hydrogen seems, at the time of writing, the best. Its efficient performance however requires to keep the ortho-hydrogen concentration as low as possible so that a catalysis system to relax the rotationally excited hydrogen molecules to the ground state will be required.

\section{Acknowledgments}

This work has been possible thanks to the support of the computing infrastructure of the i2BASQUE academic network.

Fruitful discussions with A. Takibayev (ESS), K. Batkov (ESS), M. Wohlmuther (PSI) and U. Filges (PSI) are gratefully acknowledged.

\section{References}

[1] F. Mezei, ESS Update Report, vol. Ill, Instruments and Scientific Utilisation, Technical Report, 2006.

[2] D. Filges, F. Goldenbaum, R.D. Neef, K. Nuenighoff, Ch. Pohl, E. Senitcheva, in: Proceedings of the International Collaboration on Advanced Neutron Sources, ICANS-XVI, Forschungszentrum Juelich GmbH. Doc. Number, ISSN 1433-559X, ESS Report Number ESS 03-136-M1, July, 2003, p. 579.

[3] J. Fraser et al., Neutron Production in Thick Targets Bombarded by High Energy Protons, Phys. Can., 1965.

[4] W.A. Coleman, Thermal Neutron Flux by High Energy-Protons, Report ORNLTM-2206.

[5] I.M. Thorson, et al. in: G.J. Rusell (Ed.), Proceedings of the International Collaboration on Advanced Neutron Sources, ICANS-III, Los Alamos National Laboratory, 1979, p. 31 (see also I.M. Thorson et al. Proceedings of the International Collaboration on Advanced Neutron Sources, ICANS-VII, Atomic Energy of Canada Ltd. Report AECL-888, 1984).

[6] K.F. Graham, J.M. Carpenter, Nuclear Instruments and Methods in Physics Research Section A 85 (2) (1970) 163 〈http://dx.doi.org/10.1016/0029-554X (70)90236-3).

[7] John M. Carpenter, Nuclear Instruments and Methods 145(1) 1977, p. 91 (ISSN 0029-554X, 〈http://dx.doi.org/10.1016/0029-554X(77)90560-2)).

[8] G.S. Bauer, et al., Kernforschungszentrum Karlsruhe, Report KFK- Juel-Spez113, FKF-3175, 1981.

[9] G.S. Bauer, et al. in: Proceedings of the International Collaboration on Advanced Neutron Sources, ICANS-V, Kernforschungsanlage Juelich, JuelConf-45, 1981, p. 445.

[10] J.M. Carpenter, in: Proceedings of the International Collaboration on Advanced Neutron Sources, ICANS-IV, KENS Report II, 1981, p. 105.

[11] Y. Kiyanagi, Journal of Nuclear Science and Technology 21 (1984) 735.

[12] C. Zendler, K. Lieutenant, D. Nekrassov, L. Cussen, M. Strobl, Nuclear Instruments and Methods in Physics Research Section A 704 (2013) 68.

[13] G.S. Bauer, et al. The ESS Project, vol. III, Update Report, 2003 (Chapter 4).

[14] ESS Technical Design Report, 2013 (Chapter 3, 〈http://eval.esss.lu.se/cgi-bin/ public/DocDB/ShowDocument?docid=274), Last accessed May 2013).

[15] G.S. Bauer, Atomkernenergie-Kerntechnik 41 (1982) 234.

[16] T. McManamy, M. Rennich, F. Gallmeier, P. Ferguson, J. Janney, Journal of Nuclear Materials 398 (2010) 35. (Proceedings of the Ninth International Workshop on Spallation Materials Technology).

[17] J. Carpenter, Physica B: Condensed Matter 385-386 (Part 2) (2006) 972. (Proceedings of the Eighth International Conference on Neutron Scattering).

[18] E. Shabalin, et al., Nuclear Instruments and Methods in Physics Research Section B 266 (2008) 5126.

[19] T. Kai, et al., Nuclear Instruments and Methods in Physics Research Section A $550(2005) 329$.

[20] F.X. Gallmeier, E.B. lverson, Target Moderator Reflector Assemblies for a SNS Second Target Station, Technical Report, SNS, Oak Ridge, 2009.

[21] R. Macfarlane, New Thermal Neutron Scattering Files for ENDF/B-VI Release 2 Technical Report, Los Alamos National Laboratory, 1994.

[22] M. Chadwick, et al., Nuclear Data Sheets 107 (2006) 2931. (Evaluated Nuclear Data File ENDF/B-VII.0).

[23] D. Rochman, A. Koning, Comprehensive nuclear data library with covariance data, in: JEFF Meeting, OECD/NEA, Paris, France, 2010. 
[24] S.G. Mashnik, et al., CEM03.S1, CEM03.G1, LAQGSM03.S1, and LAQGSM03.G1 Versions of CEM03.01 and LAQGSM03.01 Event-Generators, Technical Report, Los Alamos National Laboratory, March 2006.

[25] E. Iverson, Methods for Calculating the Neutronic Performance of Moderators on Pulsed Neutron Sources, unpublished.

[26] M. Magán, S. Terrón, K. Thomsen, F. Sordo, J. Perlado, F. Bermejo, Nuclear Instruments and Methods in Physics Research Section A 680 (2012) 61.

[27] T. Kai, et al., Journal of Nuclear Science and Technology 39 (2002) 120.

[28] R. MacFarlane, Cold-moderator Scattering Kernel Methods, Technical Report LA-UR-98-655, 1998.

[29] Y.Y. Milenko, R. Sibileva, Journal of Low Temperature Physics (1997).

[30] T. Kai, M. Harada, M. Teshigawara, N. Watanabe, Y. Ikeda, Nuclear Instruments and Methods in Physics Research Section A 523 (2004) 398.
[31] G. Muhrer, E. Pitcher, G. Russell, Nuclear Instruments and Methods in Physics Research Section A 536 (2005) 154

[32] G. Muhrer, M. Hartl, L. Daemen, J. Ryu, Nuclear Instruments and Methods in Physics Research Section A 578 (2007) 463.

[33] Yellow Book 〈http://www.ill.eu/fileadmin/usersfiles/OtherSites/YellowBook2008C DRom/index.htm.ILL), 2008.

[34] H. Schober, Neutron scattering instrumentation, in: L. Liang, R. Rinaldi, H. Schober (Eds.), Neutron Applications in Earth, Energy and Environmental Sciences, Neutron Scattering Applications and Techniques, Springer, USA, 2009, pp. 37-104.

[35] P. Ageron, Nuclear Instruments and Methods in Physics Research Section A 284 (1989) 197. 